\title{
DEVELOPMENT OF A PROTOTYPE CAPACITIVE BPM FOR THE UNIVERSITY OF MARYLAND ELECTRON RING*
}

\author{
$\underline{\text { Y. Zou }}^{\#}$, J.G. Wang ${ }^{+}$, Paul Chin, Y. Li, S. Bernal, M. Reiser \\ Institute for Plasma Research, University of Maryland, College Park, MD 20742
}

\begin{abstract}
Work has been performed at the University of Maryland by the electron beam physics group to design and develop a prototype capacitive Beam-Position-Monitor (BPM) for an electron ring project. Extensive theoretical work and PSPICE simulation of the equivalent circuit of the BPM have been conducted to fully understand the behaviour of the capacitive BPM. This BPM has 4 pieces of electrostatic pickups around a beam pipe of 2-inch diameter. The capacitance between the electrode and the beam pipe is approximately $0.55 \mathrm{nF}$, which results in a time constant long enough to reproduce the current waveform faithfully. A bench test of this prototype BPM has been completed. The test shows that the capacitive BPM has a very fast response to the beam current with high sensitivity. Also, the BPM can measure the beam centroid displacement with adequate accuracy. In addition, this BPM can measure the total beam current by appropriate calibration.
\end{abstract}

\section{INTRODUCTION}

Both capacitive and resistive BPMs are widely used in charged particle accelerators and beam lines. At the University of Maryland, a compact electron ring (UMER) [1-3] is being built for the study of the physics of spacecharge-dominated beams. Because the beam has relatively low energy $(10 \mathrm{keV})$, it is very easy for it to go off-center due to stray magnetic fields (the earth's field and other stray fields due to the presence of metal), misalignments, etc. To guarantee the success of the UMER, the beam centroid motion has to be measured very accurately and the information from the BPMs must be provided to a beam steering system to steer the beam back to the central orbit. Both resistive wall BPMs and capacitive BPMs will be used in the UMER. This paper will discuss the theory and design of the capacitive BPM. The resistive wall BPM has been discussed in the pervious paper.[5]

\section{BASIC PRINCIPLE}

Figures 1 and 2 are the schematics of a capacitive BPM and its equivalent circuit. In Figure 1, there are four electrodes inside the beam pipe forming four capacitors. In Figure 2, C is the capacitance between the electrode and beam pipe while $R_{1}$ is the bleeder resistor to avoid excess charge accumulation on the electrodes. The $\mathrm{R}_{1} \mathrm{C}$ time constant must be long enough to be able to reproduce the beam profile faithfully. $I_{s}$ is a frequency dependent current source. The formula to calculate $\mathrm{I}_{\mathrm{s}}$ is

$$
I_{S}=\frac{d Q}{d t}=\frac{\Phi L}{2 \pi v_{0}} \frac{d I_{b}(t)}{d t} .
$$

Here, $\mathrm{L}$ is the electrode length, $\mathrm{v}_{0}$ is the beam veolicty and $\Phi$ is the electrode angle width.

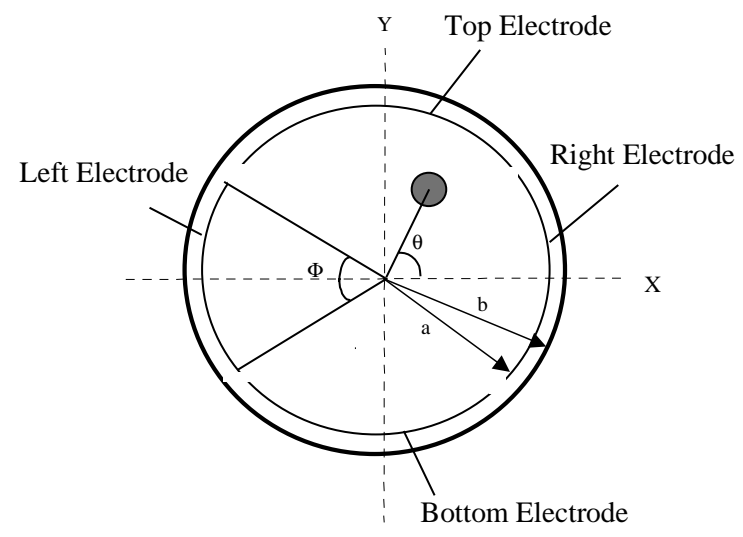

Figure 1. BPM schematic drawing.

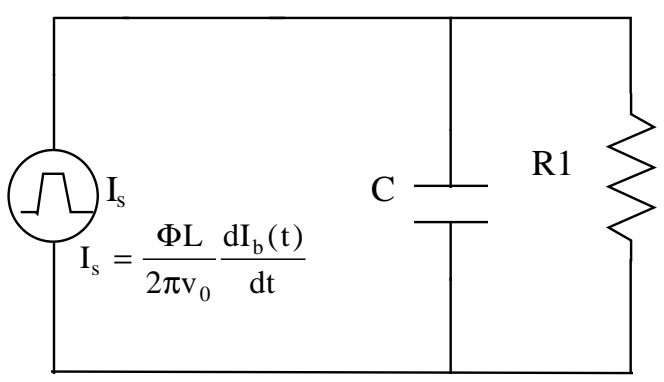

Figure 2. BPM equivalent circuit.

* Work supported by DOE.

\# Email: yunzou@glue.umd.edu

+ Current Address: ORNL and Visiting at BNL. 
If the beam is centred, the four pick-up signals are the same. When the beam is off-centred, the signals from four electrodes are different. The ratio of the signals from the two opposite electrodes will give us the beam position information. The formula is as following [4]

$$
\begin{aligned}
& 20 \log \left(V_{R} / V_{L}\right)=\frac{160}{\ln 10} \frac{\operatorname{Sin}(\Phi / 2)}{\Phi} \frac{x}{b}+\frac{20}{\ln 10}\left[\frac{8}{3} \frac{\operatorname{Sin}(3 \Phi / 2)}{\Phi}-\right. \\
& \left.16 \frac{\operatorname{Sin}(\Phi) \operatorname{Sin}(\Phi / 2)}{\Phi^{2}}+\frac{128}{3} \frac{\operatorname{Sin}^{3}(\Phi / 2)}{\Phi^{3}}\right] \frac{x^{3}}{b^{3}}+\left[-8 \frac{\operatorname{Sin}(3 \Phi / 2)}{\Phi}+\right. \\
& \left.16 \frac{\operatorname{Sin}(\Phi) \operatorname{Sin}(\Phi / 2)}{\Phi^{2}}\right] \frac{x y^{2}}{b^{3}}+\ldots
\end{aligned}
$$

If $x / b$ is small enough, we can take the first term, which is the linear term. The formula is as follows:

$$
20 \ln \left(V_{R} / V_{L}\right)=\frac{160}{\ln 10} \frac{\operatorname{Sin}(\Phi / 2)}{\Phi} \frac{x}{b} \text {. }
$$

Here, $\mathrm{b}$ is the pipe radius and $\mathrm{x}$ is beam displacement. The coefficient of the linear term is called the sensitivity of the BPM, the parameter that we are most interested in. The coefficient of the second term tells us the nonlinearity of the BPM. We can compensate this term by calibrating the $\mathrm{BPM}$ on the $\mathrm{x}$ or $\mathrm{y}$ axis. The third term, which depends on both $\mathrm{x}$ and $\mathrm{y}$ displacements, is called the coupling between the $\mathrm{x}$ and the $\mathrm{y}$ direction.

The coefficient of each term only depends on the electrode angular width $\Phi$. Both the sensitivity and the nonlinear term are greater than zero if $\Phi$ is between zero and $90^{\circ}$. The coupling term, however, could be positive or negative, depending on $\Phi$. We could let it be zero by using an appropriate electrode width $\Phi$.

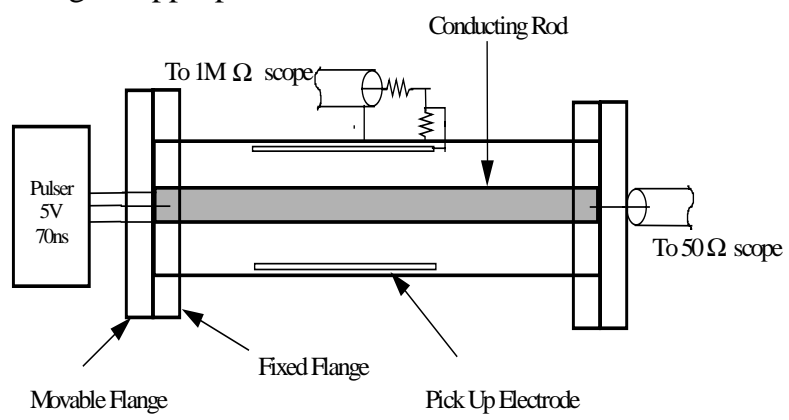

Figure 3. Bench test set up.

\section{DESIGN AND BENCH TEST}

A prototype capacitive BPM has been designed and built to test our theory of the capacitive BPM. There are four electrodes inside the pipe. The electrodes are insulated from the grounded wall by mylar, which has a dielectric constant of $2.9 \varepsilon_{0}$. Each electrode is connected to a bleeder resistor R. For the bench test, a conducting cylindrical bar was built to simulate the beam. The bar and pipe are a co-axial structure with $50 \Omega$ impedance. The bar can move freely in the radial direction inside the pipe. A pulser provides a $100 \mathrm{~mA}$ current pulse on the bar and the pulse is terminated by a $50 \Omega$ load. Figure 3 shows the set up of this bench test. The parameters of the prototype BPM are listed in Table 1.[7]

Table 1. BPM parameters

\begin{tabular}{|c|c|c|}
\hline Pipe inner radius & Electrode radius & Electrode angle \\
\hline $23.6 \mathrm{~mm}$ & $23.5 \mathrm{~mm}$ & $83^{\circ}$ \\
\hline Beam Current & Capacitance & Resistance \\
\hline $100 \mathrm{~mA}$ & $0.55 \mathrm{nF}$ & $3 \mathrm{k} \Omega$ \\
\hline
\end{tabular}

Figure 4 is a typical BPM signal from one electrode when the beam current is a rectangular waveform. This figure shows that the BPM output can reproduce the beam signal faithfully.

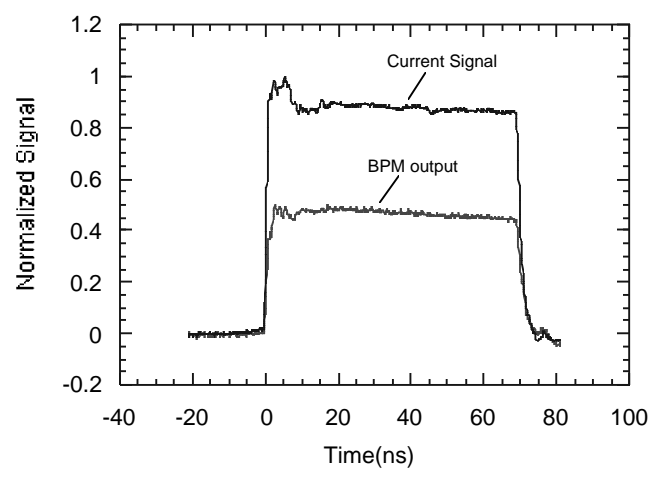

Figure 4. Typical BPM output signal.

As the beam moves off-centre, the voltage from the four electrodes are different. By calculating the voltage ratio

from two opposite electrodes we can find the displacement in the $\mathrm{X}$ and $\mathrm{Y}$ directions from Eq. 3 . However, in practice, in order to get accurate beam position measurement, the BPM has to be calibrated. Figures 5 and 6 are two calibration curves on both $\mathrm{X}$ and

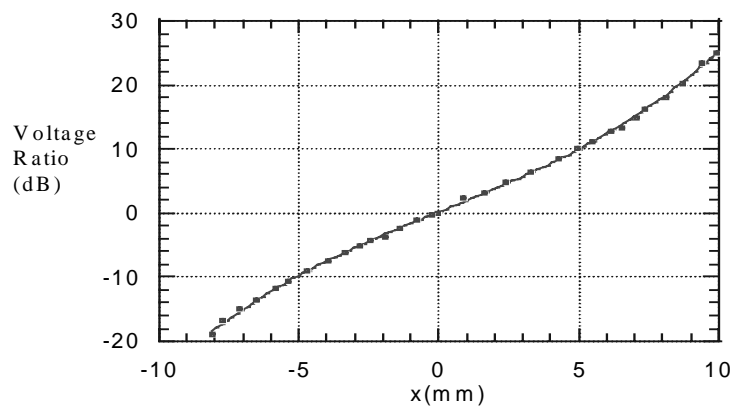

Figure 5. Calibration on $\mathrm{X}$ axis.

$\mathrm{Y}$ axes. In each figure, the horizontal axis is either $\mathrm{x}$ or $\mathrm{y}$ offset, and the vertical axis is the corresponding voltage ratio of two opposite electrodes. By using the two 
calibration curves, we assume that the voltages from the $\mathrm{X}$ and $\mathrm{Y}$ direction are not coupled, otherwise we have to calibrate the BPM on the whole X-Y plane, which is very tedious.

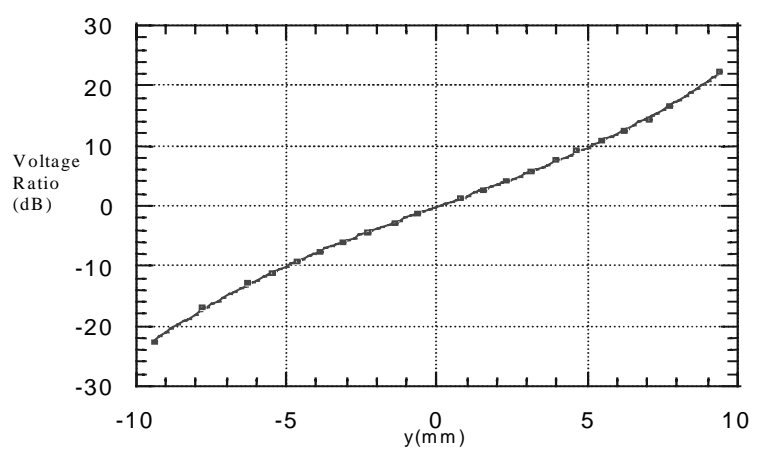

Figure 6. Calibration on $\mathrm{Y}$ axis.

To test the performance of this BPM, we have done a bench test. We set the conducting rod at different radial positions, and measured the beam positions based on the BPM signal. Then we compared the measurement and the mechanical position. Figure 7 shows the results of this measurement. In the figure, crosses are the mechanical positions and dots are BPM measurements. Within a 3$\mathrm{mm}$ displacement, measurements agree with the mechanical position pretty well (error smaller than $0.2 \mathrm{~mm}$ ). But at $6 \mathrm{~mm}$, there is a rather large difference between them (about $0.8 \mathrm{~mm}$ ). We think this is due to the coupling between $\mathrm{X}$ and $\mathrm{Y}$ electrodes. Because of the existence of the coupling term, the voltage ratio in the $\mathrm{x}$ direction depends on both $\mathrm{x}$ and $\mathrm{y}$ off-centring. We expect this systematic error will be much smaller when we optimise the electrode angle.

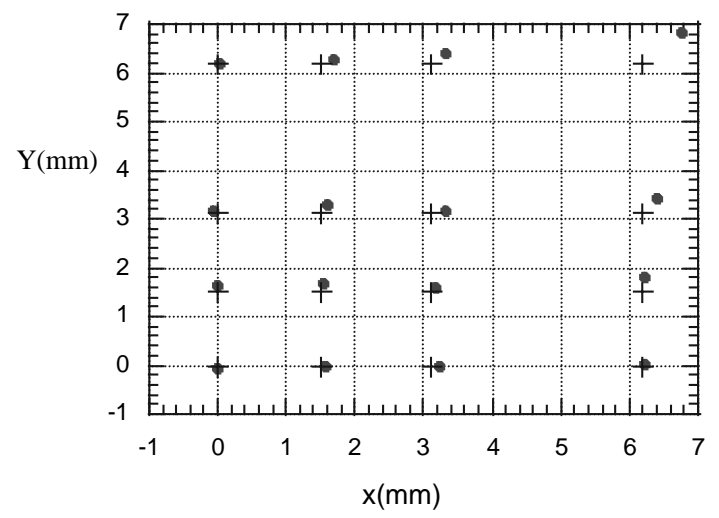

Figure 7. Comparison between the set up and measurement. (Cross: set up, Dot: measurement)

\section{CONCLUSION}

A prototype capacitive BPM has been designed and bench tested. The results show that our understanding of the capacitive BPM is correct and the performance of the BPM can meet our design objectives. A real BPM will be built and tested for electron ring.

\section{REFERENCES}

[1] M. Reiser et al, Fusion Engineering and Design, 32-33,292(1996).

[2] M. Reiser et al, Proceedings of this Conference.

[3] J.G. Wang et al., Proc. of PAC97, p. 1855 (1997).

[4] Robert E. Shafer, Beam Position Monitoring, workshop on Accelerator Instrumentation, BNL,1989.

[5] H. Suk et al., Proc. of PAC97, p. 2143 (1997).

[6] M. Reiser, Theory and Design of Charged Particle Beams, 1994, John Wiley \& Sons, INC., New York.

[7] UMER web page, http://www.ipr.umd.edu/ebte/ring. 